\title{
ARTICLE OPEN Graphene-based nanolaminates as ultra-high permeation barriers
}

Abhay A. Sagade $\mathbb{D}^{1,6}$, Adrianus I. Aria (iD ${ }^{1,2}$, Steven Edge ${ }^{3}$, Paolo Melgari ${ }^{3}$, Bjoern Gieseking ${ }^{4}$, Bernhard C. Bayer ${ }^{5}$, Jannik C. Meyer ${ }^{5}$, David Bird ${ }^{3}$, Paul Brewer ${ }^{4}$ and Stephan Hofmann (iD ${ }^{1}$

Permeation barrier films are critical to a wide range of applications. In particular, for organic electronics and photovoltaics not only ultra-low permeation values are required but also optical transparency. A laminate structure thereby allows synergistic effects between different materials. Here, we report on a combination of chemical vapor deposition (CVD) and atomic layer deposition (ALD) to create in scalable fashion few-layer graphene/aluminium oxide-based nanolaminates. The resulting $\sim 10 \mathrm{~nm}$ contiguous, flexible graphene-based films are $>90 \%$ optically transparent and show water vapor transmission rates below $7 \times 10^{-3} \mathrm{~g} / \mathrm{m}^{2} / \mathrm{day}$ measured over areas of $5 \times 5 \mathrm{~cm}^{2}$. We deploy these films to provide effective encapsulation for organic light-emitting diodes (OLEDs) with measured half-life times of $880 \mathrm{~h}$ in ambient.

npj 2D Materials and Applications (2017)1:35; doi:10.1038/s41699-017-0037-z

\section{INTRODUCTION}

Gas and water permeation barrier films play a vital part in applications ranging from food and pharmaceutical packaging to electronic devices. ${ }^{1}$ Many organic or electrode materials are highly sensitive to moisture and oxygen, hence, e.g., practically all organic optoelectronic applications such as organic light-emitting diodes (OLEDs) require efficient encapsulation. ${ }^{2,3}$ The maximum allowable permeation rates depend on the required lifetimes for each particular application and hence these rates are constantly under debate. ${ }^{1,4-6}$ For example, organic optoelectronic devices generally have the most stringent requirements, and hence are often used as benchmark application for ultra-barrier films. ${ }^{3,4} \mathrm{~A}$ common encapsulation for organic devices is a glass or metal foil which, however, is not suitable for an increasing range of applications where large-area, bendability or transparency is required. Atomic layer deposited (ALD) films such as alumina $\left(\mathrm{AlO}_{x}\right)$ are reasonably good barrier layers. Depending on the precursors used and post-deposition processing, they show water vapor transfer rates (WVTR) $<10^{-5} \mathrm{~g} / \mathrm{m}^{2} /$ day ${ }^{7-9}$ and with considerable reliability in flexibility. ${ }^{10}$ Measuring WVTR $<10^{-5} \mathrm{~g} /$ $\mathrm{m}^{2} /$ day and its quantification for commercial large area samples is a challenging task on its own to be addressed. ${ }^{5,11}$ State-of-the-art flexible thin film barrier layers are typically based on metal oxides or nitrides combinations in conjunction with polymer intermediates, with overall an thickness from hundreds of nanometers to few microns. ${ }^{12}$

2D materials, like graphene, are highly promising as ultra-high barrier materials, and their atomic thinness, mechanical and optical transparency properties offers many new possibilities and device form factors. Micron-sized single crystal graphene has been shown to be impermeable to most gases, ${ }^{13-15}$ however, over large areas $\left(>10 \mathrm{~cm}^{2}\right)$ which most applications require the scenario is completely different. While continuous and large-area mono-layer or few-layer graphene films can now be fabricated by chemical vapor deposition (CVD), ${ }^{16-18}$ such atomic films are practically prone to have defects including grain-boundaries when produced at high throughput. Recent literature on such CVD graphene films highlights that permeation through defects can be mitigated by using more layers. Yet, layer-by-layer transferred few-layer graphene stacks show WVTRs in the order of $10^{-1} \mathrm{~g} / \mathrm{m}^{2} /$ day, ${ }^{19-23}$ which is at least two orders of magnitude too high to be suitable for organic devices. Barrier membranes based on (exfoliated) graphene or graphene oxide flakes inevitably have to be much thicker to compensate for the many possible permeation pathways, ${ }^{24,25}$ which negates at least part of the advantages that $2 D$ materials can offer. It remains unclear if high-quality few-layer graphene films grown directly by CVD would show significantly lower WVTRs. The alternative is to decorate and block the defects in graphene with polymers and metal oxides. ${ }^{26-28}$ These process steps are non-trivial considering that graphene is hard to coat/wet in particular once it has been exposed to ambient air. ${ }^{29}$

Here, we combine catalytic CVD and ALD to create in scalable fashion few-layer graphene/aluminium oxide-based nanolaminates. The resulting $\sim 10 \mathrm{~nm}$ contiguous and bendable graphene-based films show water vapor transmission rates below $7 \times 10^{-3} \mathrm{~g} / \mathrm{m}^{2} /$ day measured over areas of $5 \times 5 \mathrm{~cm}^{2}$ while maintaining $>90 \%$ optical transparency. The growth of these layers is confirmed and characterized by scanning electron microscopy (SEM), transmission electron microscopy (TEM) and Raman spectroscopy mapping. We systematically benchmark the barrier performance of these graphene-based nanolaminates with existing state-of-the-art commercial multi-stacked barriers and

\footnotetext{
${ }^{1}$ Electrical Engineering Division, Department of Engineering, University of Cambridge, $9 \mathrm{JJ}$ Thomson Avenue, Cambridge CB3 0FA, UK; ${ }^{2}$ Surface Engineering and Nanotechnology Institute, School of Aerospace, Transport and Manufacturing, Cranfield University, College Road, Cranfield MK43 OAL, UK; ${ }^{3}$ National Printable Electronics Centre, Centre for Process Innovation, Thomas Wright Way, NETPark, Sedgefield TS21 3FG, UK; ${ }^{4}$ National Physical Laboratory, Hampton Rd, Teddington, Middlesex TW11 0LW, UK and ${ }^{5}$ Faculty of Physics, University of Vienna, Boltzmanngasse 5, A-1090 Vienna, Austria

Correspondence: Abhay A. Sagade (abhaysagade03@gmail.com) or Stephan Hofmann (sh315@cam.ac.uk)

${ }^{6}$ Present address: SRM Research Institute and Department of Physics \& Nanotechnology, SRM University, Kattankulathur 603203 Tamil Nadu, India
}

Received: 4 April 2017 Accepted: 11 September 2017

Published online: 23 October 2017 

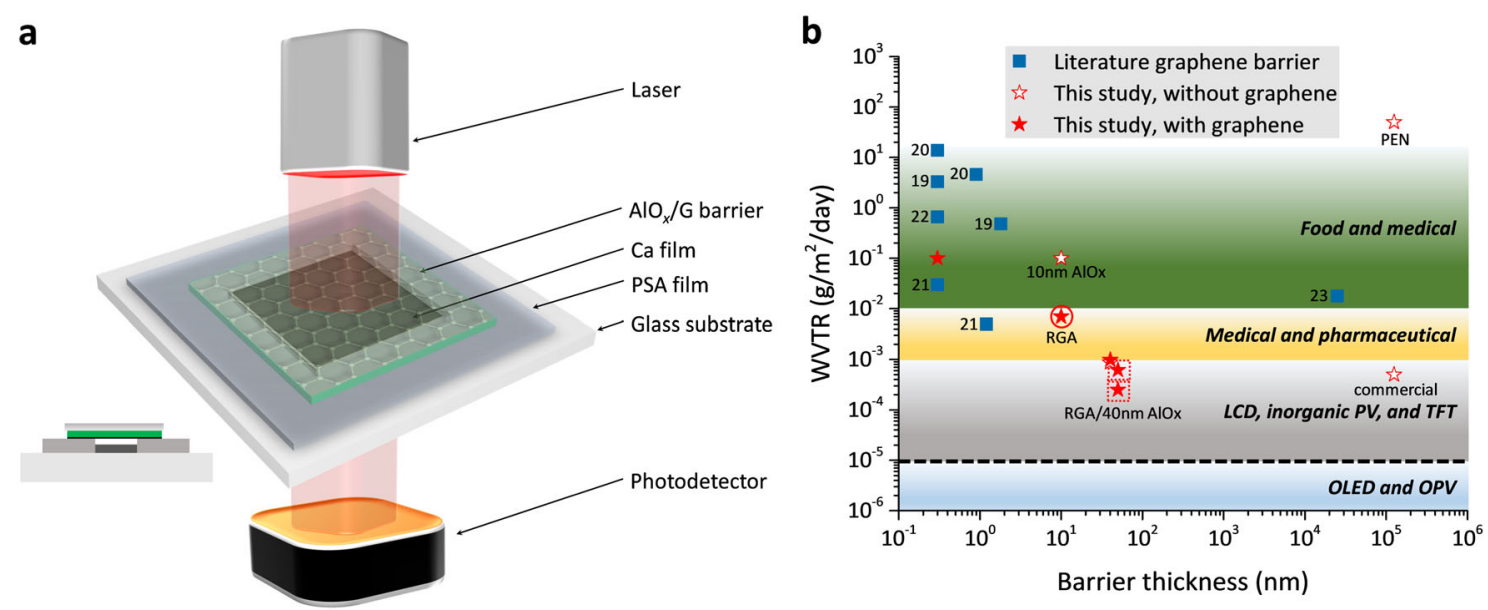

Fig. 1 WVTR measurement. a Optical Ca-test set up utilized for the WVTR measurements. The inset shows a cross-section of the final device stack. b Overview of WVTR values in context of barrier film thickness for various application requirements. The numbers next to square data points are from reported literature references, while star data points are for present study. The dashed line indicates the base value measured for comparative glass barriers fabricated in this investigation along with each batch of graphene-based barriers

thin ALD aluminium oxide $\left(\mathrm{AlO}_{x}\right)$ films. It is found that these nanolaminates show not only lower WVTRs but also more reliable, reproducible performance over large areas compared to simple sequential layering of (transferred) graphene and ALD $\mathrm{AlO}_{x}$. We demonstrate that these laminates encapsulate OLEDs reasonably well with measured half-life times of $880 \mathrm{~h}$ in ambient.

\section{RESULTS}

While WVTRs and barrier performance can be characterized by a range of techniques ${ }^{1,4}$ including MOCON, electrical and optical $\mathrm{Ca}$ tests, etc. for each of them sample compatibility and preparation can be a challenge. In particular, for novel material systems like graphene, reliability and accuracy of the measurements is nontrivial. For meaningful measurements of low WVTRs, here we use two independent techniques with low detection limits, namely optical monitoring of Ca film thickness (Fig. 1a) and traceability measurements based on cavity ring down spectroscopy (Supplementary Fig. 1), as described in the methods section. All results refer to a barrier film size of $5 \times 5 \mathrm{~cm}^{2}$, handled and transferred in air unless otherwise stated. As reference, we measure WVTRs of $\mathrm{AlO}_{x}$ films of various thicknesses grown by ALD (see methods) on a polyethylene naphthalate (PEN) substrate. The ALD recipe is optimized to obtain a highly uniform, good quality film with a refractive index of 1.65, indicative of high density alumina required for barrier applications. ${ }^{8}$ These alumina films are used without further post-treatments such as annealing or $\mathrm{O}_{2}$ plasma. We measure WVTRs for 10 and $40 \mathrm{~nm} \mathrm{AlO} / \mathrm{PEN}$ films to be 0.1 and $8.7 \times 10^{-4} \mathrm{~g} / \mathrm{m}^{2} /$ day, respectively, at $22^{\circ} \mathrm{C} / 50 \% \mathrm{RH}$ (relative humidity). Lower WVTR values have been reported for $\mathrm{AlO}_{x}$, but only for the alumina layer directly deposited on $\mathrm{Ca}$ by in situ glovebox production lines without exposing to ambient. ${ }^{8}$ Figure $1 \mathrm{~b}$ provides an overview of WVTR values in the context of barrier film thickness for various application requirements and measured in this study (see Supplementary Table 1 for details on all samples).

Figure $2 \mathrm{a}$ is a schematic overview of the process flow used for nanolaminate fabrication. Continuous monolayer graphene is grown by CVD (see methods) and transferred via a typical wet chemical process with PMMA. In the following we refer to this as standard process (Fig. 2a). The growth parameters are optimized to obtain a large average graphene grain size of $100-200 \mu \mathrm{m}$, which (to our knowledge) is larger than any graphene barrier sample reported previously (see Supplementary Table 1). WVTR of such monolayer graphene (G/PEN) is measured to be $0.1 \mathrm{~g} / \mathrm{m}^{2}$ / day. While this value is comparable to previous reports, ${ }^{20,22}$ it should be noted that here our samples $\left(25 \mathrm{~cm}^{2}\right)$ are more than 6 times larger in area $\left(4 \mathrm{~cm}^{2}\right)$. Our results show that monolayer graphene and $10 \mathrm{~nm}$ of ALD alumina have similar WVTRs. Regarding further relevant reference samples, we find that neither a $40 \mathrm{~nm}$ ALD alumina coating on monolayer G/PEN nor a monolayer graphene layer transferred onto a $40 \mathrm{~nm} \quad \mathrm{AlO}_{x} / \mathrm{PEN}$ sample lead to any significant barrier performance improvement relative to the plain $40 \mathrm{~nm} \mathrm{AlO}_{x} / \mathrm{PEN}$ reference. We relate this to defects in the graphene films being not sufficiently decorated/ blocked as well as to the well-known challenge of reliably interfacing with graphene. A similar observation has been made recently by Nam et al. ${ }^{30}$ Also, we find that the results delicately depend on process (e.g., growth and transfer) details and hence we found it difficult to develop a reliable process based on this standard route.

We therefore establish here a modified growth process as highlighted in Fig. 2a. After the initial graphene CVD, we deposit a $10 \mathrm{~nm} \mathrm{AlO}_{x}$ film while the graphene is still on the $\mathrm{Cu}$ growth substrate $(\mathrm{G} / \mathrm{Cu})$. We then form a nanolaminate structure by carrying out another graphene CVD run onto the $\mathrm{AlO}_{x} / \mathrm{G} / \mathrm{Cu}$ stack. The latter we refer to as second (2nd) growth and the resulting structure as re-grown $\mathrm{AlO}_{x}$ /graphene (RGA), see Supplementary Fig. 2. In order to assure the initial ALD $\mathrm{AlO}_{x}$ coating to be as uniform as possible, we use here a ALD process with 10 water prepulses, ${ }^{28,31}$ which assists the nucleation of $\mathrm{AlO}_{x}$. Figure $3 \mathrm{a}$, b shows SEM images of the as-deposited $10 \mathrm{~nm} \mathrm{AlO}_{x}$ film on $\mathrm{G} / \mathrm{Cu}$. The $\mathrm{AlO}_{x}$ layer is seen to form continuously all over and not selectively at grain boundaries or defects. ${ }^{27,28}$ Figure 3 c shows cross-sectional high-resolution TEM (HRTEM) analysis of the RGA structure. The 2nd growth leads to additional graphene layer formation beneath the $\mathrm{AlO}_{x}$ at the existing $\mathrm{G} / \mathrm{Cu}$ interface, while the $\mathrm{AlO}_{x}$ film appears to maintain its thickness. The thickness of the resulting graphene film is $\sim 1 \mathrm{~nm}$, indicating 3-4 layers of graphene. Plan-view TEM and selected area electron diffraction (SAED) measurements of suspended nanolaminates reveal that during the 2nd CVD step the initially amorphous $\mathrm{ALD} \mathrm{AlO}_{x}$ layer transforms into a nanocrystalline film (Fig. 3d and Supplementary Fig. 3). Interestingly, the SAED pattern of this nanocrystalline film is not indicative of a crystalline $\mathrm{Al}_{2} \mathrm{O}_{3}$ phase but best matched to the rarely reported $^{32,33}$ Al-oxycarbide $\mathrm{Al}_{2} \mathrm{OC}$. This suggests structural 
a

Standard process

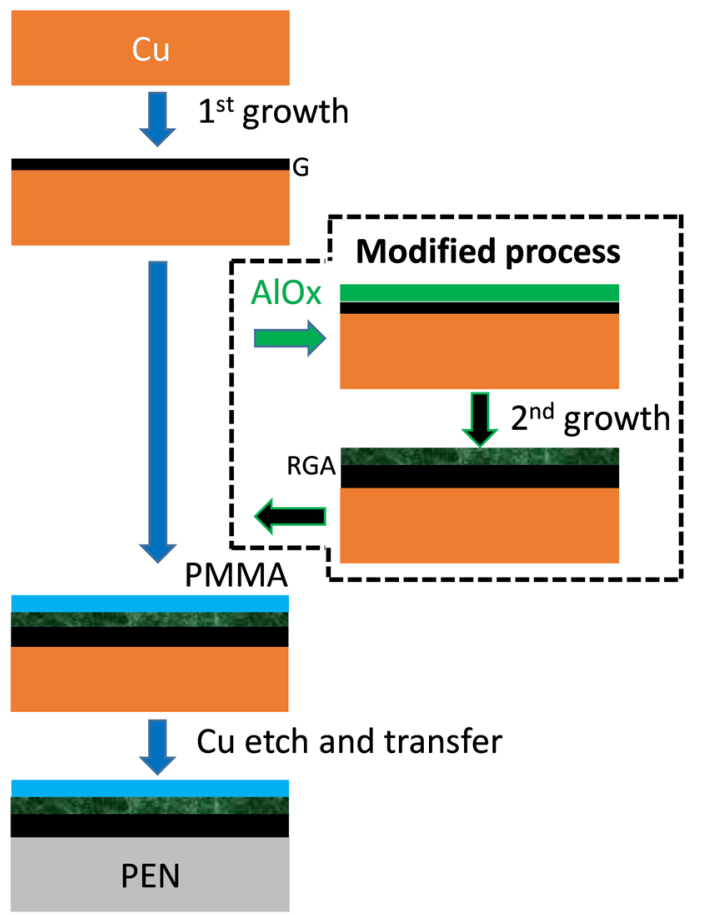

b

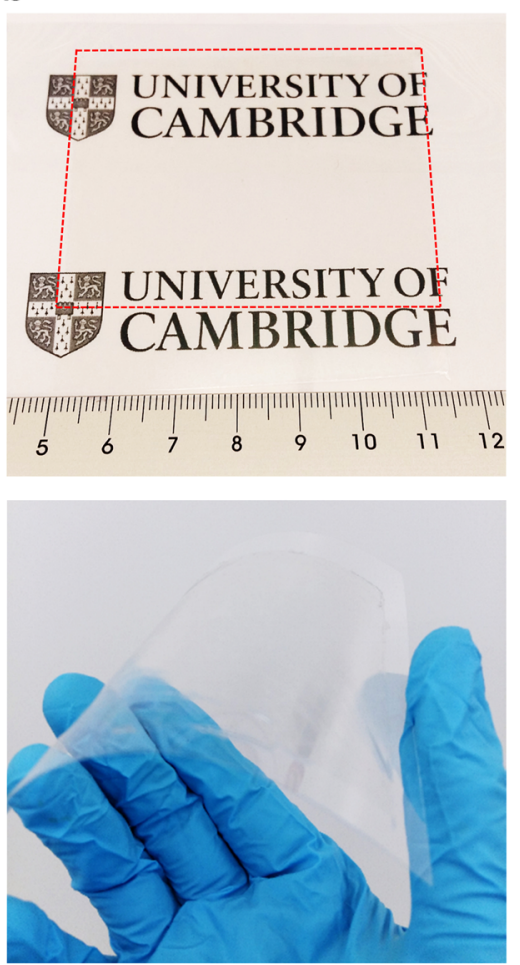

Fig. 2 Nanolaminate process. a Schematic of the process flow used for graphene and laminate samples. b Optical photographs of large area $7 \times 7 \mathrm{~cm}^{2}$ RGA film on PEN substrate highlighting transparency and bendability

changes in the $\mathrm{AlO}_{x}$ film upon the 2nd growth step, i.e. Aloxycarbide formation, hence here-after we refer to the nanocrystalline phase in the RGA film as 'AlO ${ }_{x}$-based film'. We emphasize that carrying out a second growth step without the alumina, i.e. just on the $\mathrm{G} / \mathrm{Cu}$, does not lead to the repair of graphene defects, rather at the given CVD conditions destroys the first graphene layer during the $\mathrm{H}_{2} / \mathrm{Ar}$ annealing step or during growth. Hence, the process order is important to achieve synergistic effects between the different materials (See Supplementary Figs. 4 and 5). In the given sequence, the process promotes active bonding/ interaction between the graphene and $\mathrm{AlO}_{x}$-based film (Fig. 3d). In our modified process the $10 \mathrm{~nm} \mathrm{AlO}_{x}$ layer serves multiple purposes: (i) it acts as protecting layer to the monolayer graphene during the second growth, (ii) it (initially being amorphous and not too thick) allows permeation of carbonaceous species for the subsequent second graphene growth at high temperature, (iii) after the second growth it acts as part of an effective nanolaminate structure, and iv) it also acts as mechanical support in the handling of large-area graphene. Figure $2 \mathrm{~b}$ shows optical images of such $\sim 10 \mathrm{~nm}$ thin RGA layer of $7 \times 7 \mathrm{~cm}^{2}$ in size, transferred with PMMA on a PEN substrate. The sample is $>90 \%$ optically transparent (see Supplementary Fig. 6) and easily bendable (Supplementary Video 1).

The 1st and 2nd growth samples are further probed by Raman spectroscopy after transfer onto $300 \mathrm{~nm} \mathrm{SiO} 2 / \mathrm{Si}$ substrates (Fig. 4). For the 1 st growth, the Raman spectra show an intensity ratio $I_{2 \mathrm{D} / \mathrm{G}}$ of $\sim 2-3.7$ with a small $D$ peak $\left(I_{D / G}<0.15\right)$ (see Supplementary Fig. 7) indicative of good quality monolayer graphene. The uniformity of the growth is highlighted by the $I_{2 \mathrm{D} / \mathrm{G}}$ map in Fig. $4 \mathrm{~b}$. On the other hand, RGA samples show an $I_{2 \mathrm{D} / \mathrm{G}}$ ratio of $\sim 0.74-1.8$ and $I_{\mathrm{D} / \mathrm{G}}$ $<0.1$, consistent with the formation of 2-3 layers of graphene without additional damage. This is further evidence that the regrowth process results in few layers of graphene underneath the $\mathrm{AlO}_{x}$ film on the parent catalytic $\mathrm{Cu}^{34-36}$ As we here focus on the barrier layer application, a detailed study of the growth mechanisms of such buried interface graphene is beyond the scope of this work.

As a simple initial test of the permeation properties of the RGA films, we heated them on a hot plate at $200^{\circ} \mathrm{C}$ in ambient while still on the $\mathrm{Cu}$ support along with only monolayer $\mathrm{G} / \mathrm{Cu}$ and bare $\mathrm{Cu}$ foil (Supplementary Fig. 8). The level of $\mathrm{Cu}$ oxidation will then directly reflect the barrier properties of the films. While for $\mathrm{Cu}$ and $\mathrm{G} / \mathrm{Cu}$ samples a reddish color is observed within 15-20 s and 30-35 s, respectively, indicative of a rapid Cu oxidation, the RGA/ Cu samples do not show any sign of oxidation even after $30 \mathrm{~min}$ of heating. This is an immediate and positive indication that the RGA nanolaminate is uniform and pin-hole free and can act as highquality moisture barrier.

In contrast to previous reports on graphene barrier films which aimed at clean graphene layers with minimal polymer contamination, ${ }^{19,20}$ here for WVTR measurement study we do not remove the PMMA layer and utilize it as: (a) protection during Ca oxidation since $\mathrm{Ca}$ is known to form micron scale protrusions which can penetrate through the barrier film and damage it, ${ }^{37,38}$ (b) insulating layer when used as encapsulation coating for electronic devices separating the conductive graphene, and (c) ease of sample handling. We note that the WVTR of $0.3-\mu \mathrm{m}$ thick PMMA is several orders higher, hence it will not affect on the measured values of graphene-based laminates. In WVTR measurement via the optical Ca test (see Fig. 1a), the spot size of used laser is large enough to cover $\mathrm{cm}^{2}$ area of sample rather a small spot around the defects. Hence, the WVTR values correspond to a practical average over the nanolaminate samples. The surface topography of these nanolaminate samples is smooth with a measured rms roughness $<10 \mathrm{~nm}$ (Supplementary Fig. 9). The RGA sample of $\sim 10 \mathrm{~nm}$ thickness showed a WVTR of $7 \times 10^{-3} \mathrm{~g} / \mathrm{m}^{2} /$ day at $22^{\circ} \mathrm{C} /$ $50 \% \mathrm{RH}$. This value is two orders better than the monolayer and other graphene-based moisture barriers reported in literature for large sample sizes (see Fig. $1 \mathrm{~b}$ and Supplementary Table 1). While the simple addition of monolayer graphene did not improve the 


\section{$1^{\text {st }}$ growth}

a

b

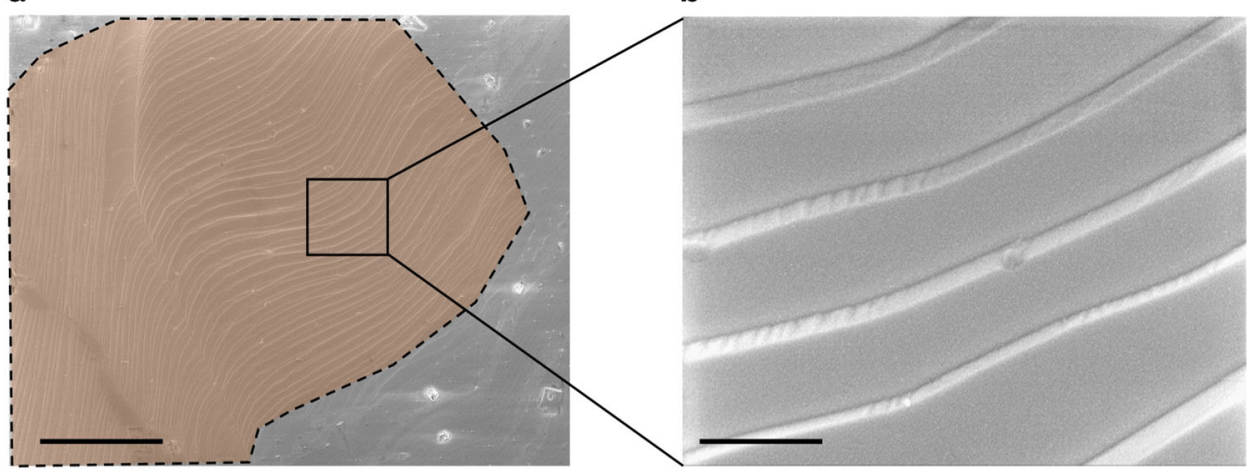

$2^{\text {nd }}$ growth

C
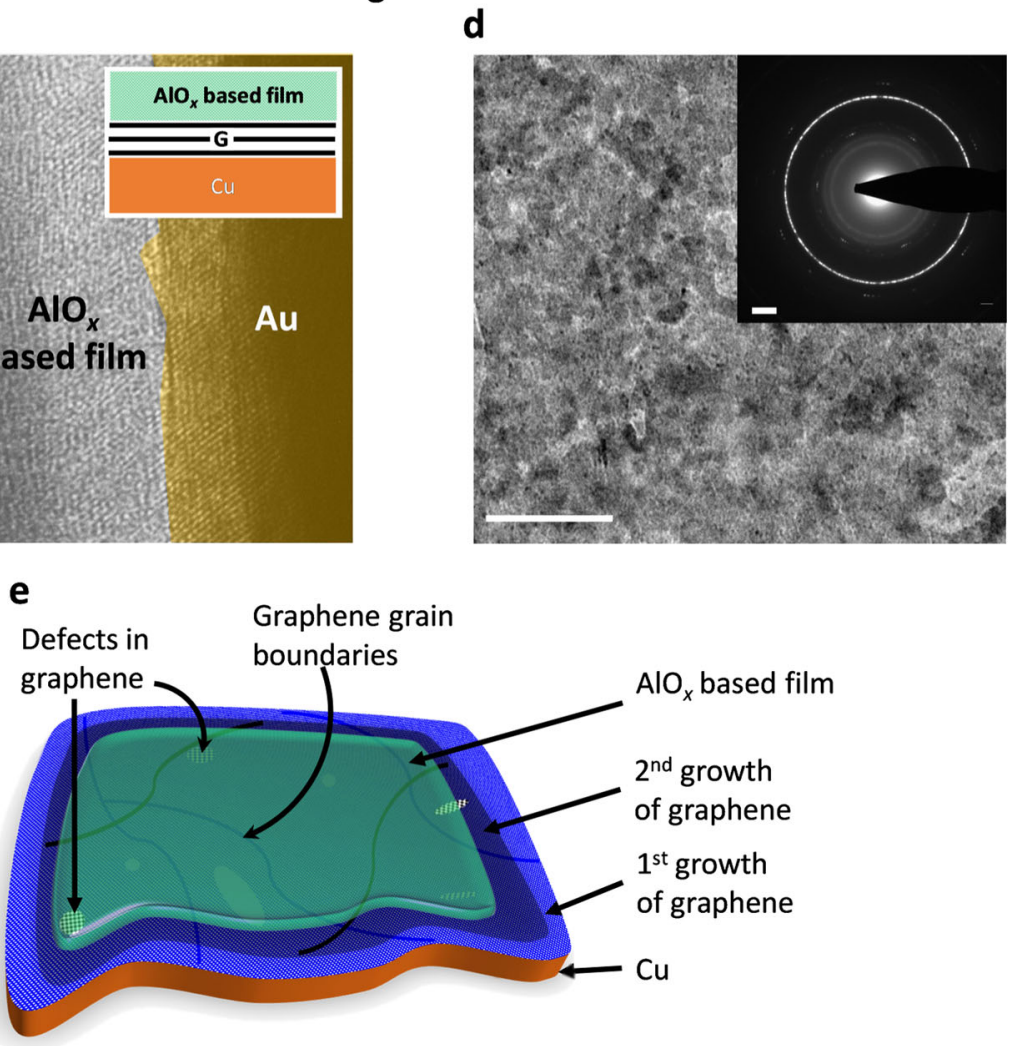

Fig. 3 Mircoscopy of graphene barrier films. a SEM image of continuous growth of $10 \mathrm{~nm} \mathrm{AlO}_{x}$ on a single graphene grain (dotted area) on Cu foil after 1st growth and a representative magnified area in (b). The cross-sectional HRTEM image in c shows the presence of few layer graphene below the $\mathrm{AlO}_{x}$-based layer after the 2nd growth, as illustrated in the inset. d Bright field TEM image of a suspended RGA film in plan-view, showing the nanocrystalline nature of the $\mathrm{AlO}_{x}$-based phase after the 2nd growth step. The inset shows the corresponding SAED pattern, which consists of sets of hexagonally arranged spots assigned to few layer graphene and a ring pattern corresponding to a nanocrystalline phase of the Al-oxycarbide $\mathrm{Al}_{2} \mathrm{OC}$, as described in Supplementary Fig. 3. e Schematic highlighting the nanolaminate structure and coverage of graphene defects by $\mathrm{AlO}_{x}$-based layer. The scale bar in $\mathbf{a}$ is $10 \mu \mathrm{m}, \mathbf{b}$ is $0.5 \mu \mathrm{m}, \mathbf{c}$ is $5 \mathrm{~nm}$, and $\mathbf{d}$ is $100 \mathrm{~nm}$

barrier properties of the $\mathrm{AlO}_{x}$ (see above), ${ }^{30}$ the transfer of one PMMA/RGA layer on $40 \mathrm{~nm} \mathrm{AlO}_{x} / \mathrm{PEN}$ showed 3 times improved performance with a measured WVTR of $2.5 \times 10^{-4} \mathrm{~g} / \mathrm{m}^{2} /$ day (see Supplementary Fig. 10 and Table1).

To explore the possibility of multi-stack laminates consisting of RGA and $\mathrm{AlO}_{x}$, we focus on a $20 \mathrm{~nm} \mathrm{AlO}$ / RGA/20 nm $\mathrm{AlO}_{x} / \mathrm{PEN}$ structure. Further, we compare the Ca test WVTR values to measurements in the traceability set up based on cavity ring down spectroscopy. This sandwiched laminate showed a WVTR of $4.4 \times$ $10^{-3} \mathrm{~g} / \mathrm{m}^{2} /$ day at $38^{\circ} \mathrm{C} / 90 \% \mathrm{RH}$ in the Ca test. In comparison, the traceability measurement for such sample gave a value of $6.02 \times$
$10^{-4} \mathrm{~g} / \mathrm{m}^{2} /$ day. The difference might be partly related to the distinct sample handling during preparation. This traceability result is similar to results for $125-\mu$ m thick commercial barrier films which we used as benchmark (multi-stacked layers with few microns of mechanical protections; see Fig. $1 \mathrm{~b}$ and Supplementary Table 1). In principle, the key mechanism of WVTR improvement in the laminates is blocking the moisture paths, therefore sandwiching one RGA layer between two $\mathrm{AlO}_{x}$ layers $\left(\mathrm{AlO}_{x} / \mathrm{RGA} / \mathrm{AlO} \mathrm{O}_{x}\right)$ is expected to show improved barrier properties.

In order to validate the adaptability of the introduced RGA barrier films, we use them here to encapsulate standard OLED 
a

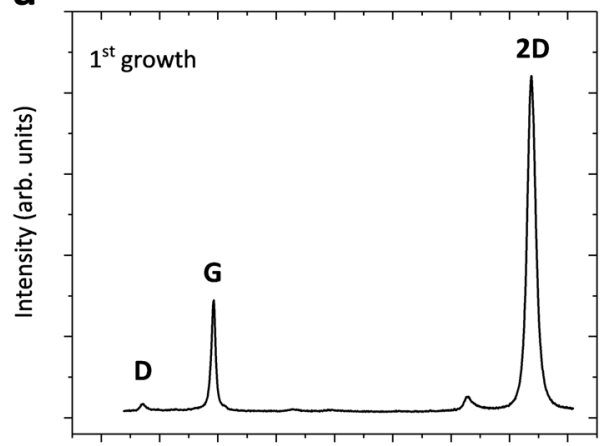

120014001600180020002200240026002800

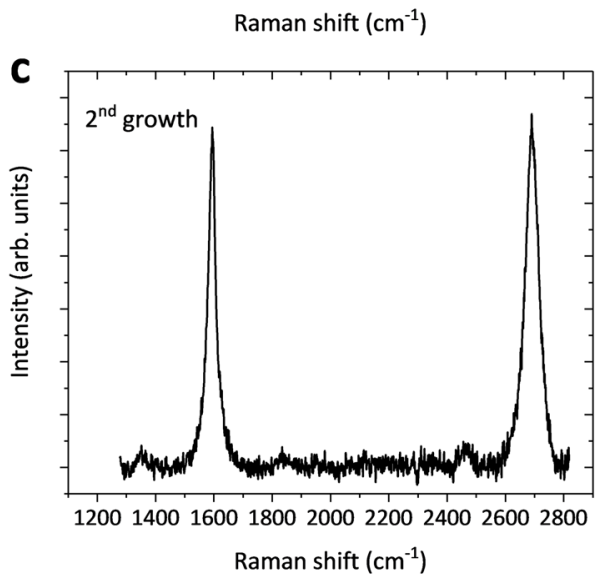

b
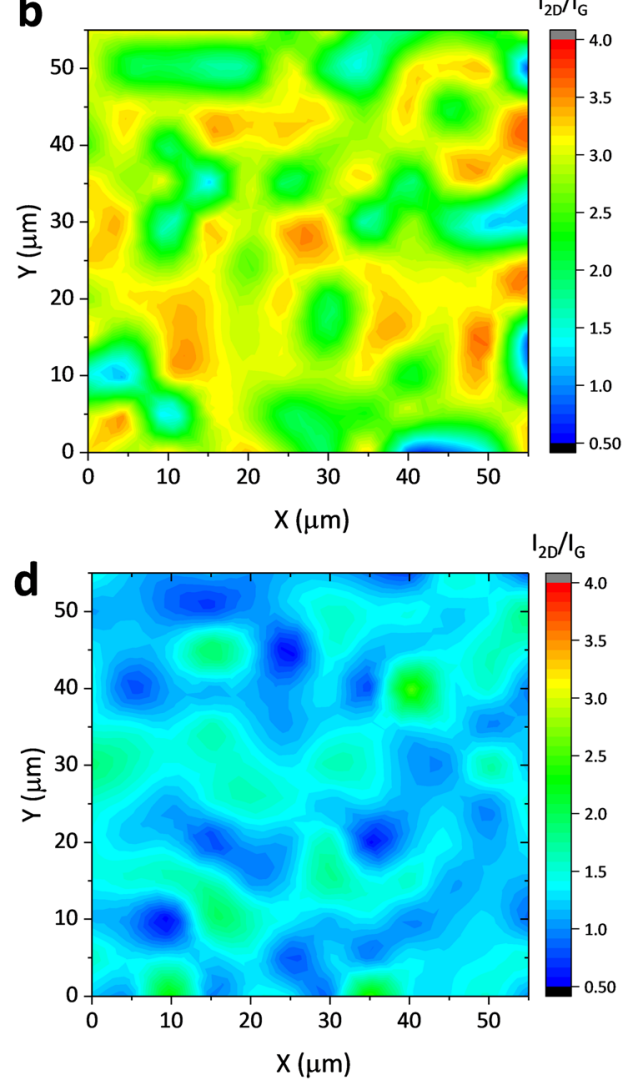

Fig. 4 Spectroscopy of graphene barrier films. Raman spectroscopy of graphene after first (a,b) and second (c,d) growth

devices. The OLED stack (see methods) was made on glass substrate and subsequently a PMMA/RGA/PEN barrier was laminated onto it without using any additional mechanical protection layers, as depicted in Fig. $5 \mathrm{a}-\mathrm{c}$ and $\mathrm{d}$ show optical images of a $1 \times 1 \mathrm{~cm}^{2}$ glowing OLED in ambient, as-fabricated and after 1 week, respectively. For comparison, we also measured two more samples with $40 \mathrm{~nm} \mathrm{AlO} x / \mathrm{PEN}$ and a commercial barrier of $125 \mu \mathrm{m}$ (as studied previously). The OLEDs were lit at $3000 \mathrm{~cd} / \mathrm{m}^{2}$ with $12 \mathrm{~V}$ bias and the luminescence measured over time to extrapolate the half-life time. The observed half-life times of the $40 \mathrm{~nm} \mathrm{AlO}_{x}$ and commercial barrier of $125 \mu \mathrm{m}$ are $925 \mathrm{~h}$ and 1400 $\mathrm{h}$, respectively, while it is $880 \mathrm{~h}$ for $\sim 10 \mathrm{~nm}$ RGA, demonstrating the successful integration of the transparent RGA structure as effective moisture barrier layer.

\section{DISCUSSION}

Here, we introduce a nanolaminate structure to improve on the shortcomings and challenges of effective large-area barrier formation via regular sequential layering of (transferred) graphene reported previously. These reports have highlighted the challenges of controlled "layering" of transferred graphene including the deleterious effect of polymer contamination. ${ }^{19,20}$ A number of different processes to improve the performance of polymer-based barrier layers via the addition of graphene have been reported, ${ }^{39}$ while O'Hern et al. ${ }^{26}$ have used polymer and ALD oxide deposition to "seal" practically unavoidable defects in larger-area monolayer graphene for nanofiltration applications. However, for barrier applications, in order to achieve low WVTRs the defects/pinholes in graphene cannot be effectively patched by polymers that show inherent high WVTRs. Hence, a number of reports have looked at the potential of ALD oxides, particularly $\mathrm{AlO}_{x}$, to decorate grain boundaries and defects in graphene. ${ }^{27,30}$ Nevertheless, effective "sealing" of larger holes in graphene with a simple ALD coverage is challenging. In line with this previous literature, we show that neither an ALD alumina coating onto graphene nor graphene transferred onto a $\mathrm{AlO}_{x}$ film are reliable and effective means to straightforwardly achieve synergistic effects to significantly enhance barrier performance over practically required large areas. This is due to a range of reasons, including damage of the ALD oxide layer during subsequent transfer and poor adhesion of ALD oxide layers on the graphene.

Our new route of forming a nanolaminate structure by carrying out another graphene CVD run (2nd growth, Fig. 2a) onto an $\mathrm{AlO}_{x} /$ $\mathrm{G} / \mathrm{Cu}$ structure effectively leads to further graphene layer growth fed by precursor permeation through the existing film. We speculate that important thereby are not only the additional graphene layers but also the "self-formation" of a compact and contiguous structure combining the graphene and $\mathrm{AlO}_{x}$-based film. This is also indicated by the suggested phase transformation of the initially amorphous $\mathrm{AlO}_{x}$ layer towards a nanocrystalline $\mathrm{AlO}_{x}$-based oxycarbide upon the 2nd CVD step (Supplementary Fig. 3). Such RGA layers show WVTRs of $7 \times 10^{-3} \mathrm{~g} / \mathrm{m}^{2} /$ day with optical transparency of $>90 \%$ while being only $\sim 10 \mathrm{~nm}$ thin, which is significantly better than values reported in previous literature (see Fig. $1 \mathrm{~b}$ and Supplementary Table 1). This manifests proof-ofthe-concept effective heterogeneous interfacing. The introduced layer structure could be used as repeat layer unit or as part of a further multi-layer structure analogue to current commercial barrier films. Therefore, not only permeation but also a range of other properties can be tuned including adhesion and haze. We demonstrate one of such a possibility to form multi-layer stacks (of $50 \mathrm{~nm}$ ) by sandwiching RGA layer between two $20 \mathrm{~nm}$ thin $\mathrm{AlO}_{x}$ layers which shows superior barrier properties with a WVTR of $6.02 \times 10^{-4} \mathrm{~g} / \mathrm{m}^{2} /$ day, comparable to values for commercial barrier layers of $125 \mu \mathrm{m}$ thickness. While our approach is scalable and 
a

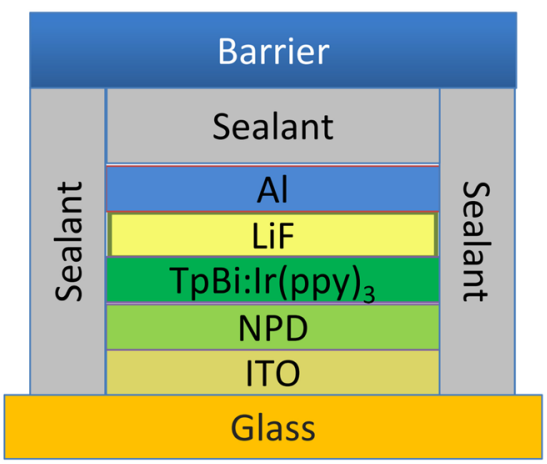

C

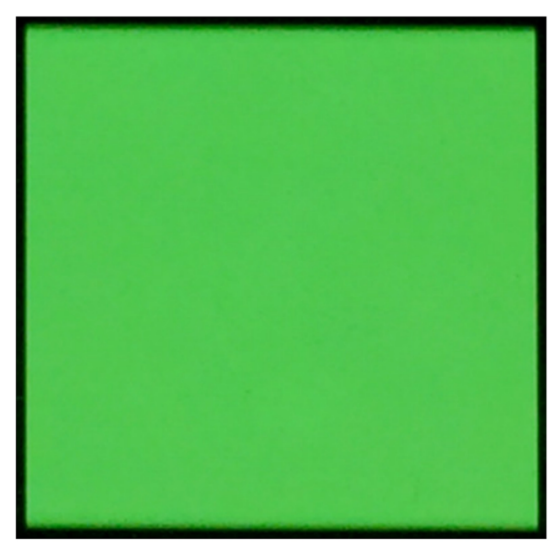

b

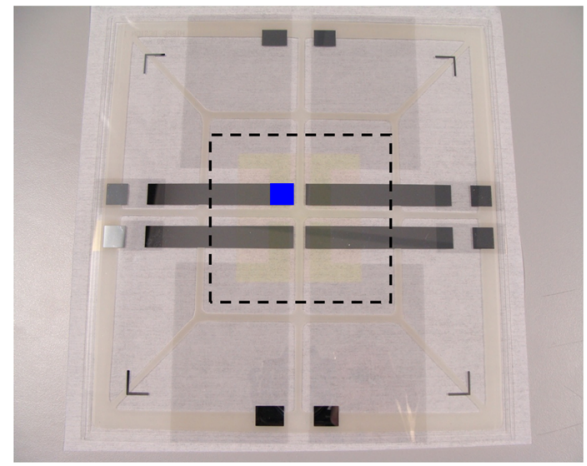

d

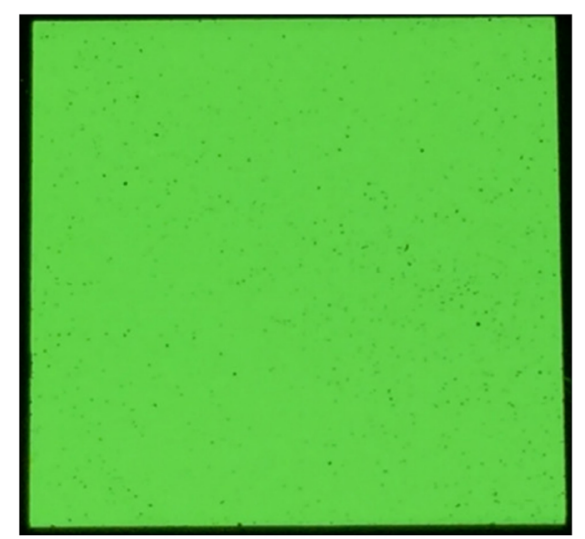

Fig. 5 OLED fabrication and encapsulation with RGA barrier. a Schematic of stacks of organic layers in OLED and $\mathbf{b}$ final device. Dotted region shows the RGA barrier of $7 \times 7 \mathrm{~cm}^{2}$ and blue box indicates the position of active OLED. Demonstration of glowing OLED of $1 \times 1 \mathrm{~cm}^{2}$ area for the endurance test in ambient: $\mathbf{c}$ just after fabrication and $\mathbf{d} 1$ week later

more error-tolerant than previous approaches, high throughput manufacturing and handling of very large area ultra-thin films still requires further innovation to assure high yield (see Supplementary Fig. 11). In summary, by combining catalytic CVD and ALD we have demonstrated $>90 \%$ transparent, contiguous and bendable graphene-based nanolaminate barrier films that show WVTRs below $7 \times 10^{-3} \mathrm{~g} / \mathrm{m}^{2} /$ day while being $\sim 10 \mathrm{~nm}$ thin and manually handled in ambient. We systematically benchmarked these WVTRs compared to existing literature and state-of-the-art barrier films and demonstrated that the nanolaminate films can be effectively integrated in OLEDs enabling half-life times of $880 \mathrm{~h}$ in ambient. These results highlight the potential of such heterogeneous material integration and the use of such nanolaminates as building block to engineer new functionalities and form factors.

\section{MATERIALS AND METHODS}

\section{Growth}

Graphene growth was carried out by chemical vapor deposition (CVD) on copper metal catalyst in a hot-wall reactor. ${ }^{29} \mathrm{~A}$ polycrystalline $\mathrm{Cu}$ foil (Alfa Aesar, $25 \mu \mathrm{m}$ thick, $99.999 \%$ purity) of $8 \times 8 \mathrm{~cm}^{2}$ was blown dry with $\mathrm{N}_{2}$ to remove any dust particles and used for electrochemical polishing (EP). For $E P$, a mixture of $\mathrm{H}_{3} \mathrm{PO}_{4}$ and water was used $(7: 3)$ as electrolyte and a constant voltage of $2.7 \mathrm{~V}$ was applied between cathode and anode for 8 $\mathrm{min}$. After polishing, the foil was rinsed for few minutes, dipped in IPA for 5 min and then dried under $\mathrm{N}_{2}$ flow. The CVD parameters for the 1st and 2nd growth (see Fig. 2a) were optimized to obtain complete coverage of the foil:

1st growth: annealing- $\mathrm{H}_{2}: \mathrm{Ar}:: 200: 50 \mathrm{sccm}$ for $90 \mathrm{~min}$, growth- $\mathrm{H}_{2}: \mathrm{Ar}$ : $\mathrm{CH}_{4}:: 35: 200: 15 \mathrm{sccm}$ for $90 \mathrm{~min}$.

2nd growth: annealing- $\mathrm{H}_{2}: A r:: 200: 50 \mathrm{sccm}$ for $60 \mathrm{~min}$, growth- $\mathrm{H}_{2}: A r$ : $\mathrm{CH}_{4}: 35: 200: 15 \mathrm{sccm}$ for $90 \mathrm{~min}$.
The growth temperature was $970{ }^{\circ} \mathrm{C}$. For the growth, $\mathrm{CH}_{4}$ at $0.1 \%$ dilution in $\mathrm{Ar}$ was used to obtain a low nucleation density leading to an larger average graphene grain size. ${ }^{40} \mathrm{~A}$ chamber pressure of $50 \mathrm{mbar}$ was maintained throughout the process.

For the re-growth, $10 \mathrm{~nm} \mathrm{AlO}$ was deposited on the $\mathrm{G} / \mathrm{Cu}$ using a Beneq TFS 200 atomic layer deposition (ALD) tool. The precursors for Al and oxygen were TMA and water, respectively, set at $300 \mathrm{sccm}$ flow rate. A conformal growth of $\mathrm{AlO}_{x}$ on $\mathrm{G} / \mathrm{Cu}$ was optimized by utilizing few water pulses which act as seed layer for the cyclic growth of the oxide..$^{28,31}$

\section{Transfer}

The grown layers were transferred on planarized $125 \mu \mathrm{m}$ thick PEN substrate (Teijin DuPont Films ${ }^{\top \mathrm{M}}$, roughness $<2 \mathrm{~nm}$ ) with standard PMMAbased wet transfer method. ${ }^{41}$

\section{Imaging and measurements}

The cross-sectional HRTEM imaging was performed on a FEI Philips Tecnai 20. Plan-view TEM and SAED of suspended nanolaminates were done in a Philips CM200 at 80 kV electron acceleration voltage. Raman spectra were recorded using a Renishaw system at $532 \mathrm{~nm}$ wavelength.

The WVTR was calculated using Ca test. A corning glass of $10 \times 10 \mathrm{~cm}^{2}$ was cleaned and dried in antechamber of glovebox along with graphene samples at $60^{\circ} \mathrm{C}$ overnight. The pressure-sensitive adhesive sealant was applied on the glass with a centre opening of $3 \times 3 \mathrm{~cm}^{2}$. A $100 \mathrm{~nm}$ Ca film was evaporated in the centre using a shadow mask and then the G/PEN sample was laminated gently across the Ca film. The quantitative study of permeation was performed by monitoring optical transmission of the glass/Ca/G/PEN stack. A rectangular laser beam $\left(0.5 \times 2 \mathrm{~cm}^{2}\right)$ of wavelength $630 \mathrm{~nm}$ was used. The optical transmission of the cell was measured and analysed using Beer-Lambert's law to calculate the WVTR values. Unless mentioned, these measurements were carried out at $22^{\circ} \mathrm{C}$ and $50 \% \mathrm{RH}$. Accelerated tests were also performed for selected samples at $38^{\circ} \mathrm{C}$ and 
$90 \%$ RH. Traceability measurements were performed at the NPL facility with a custom-built set-up based on cavity ring down spectroscopy ${ }^{6}$ (see Supplementary Fig. 1). In addition, MOCON Aquatran-2 measurements have been carried out for selective reference samples.

\section{OLED fabrication}

The OLED devices were PHOLED (phosphorescent OLED) with a structure consisting of ITO $(100 \mathrm{~nm}) / \mathrm{NPD}(100 \mathrm{~nm}) /(\mathrm{TpBi}: \mathrm{Ir}(\mathrm{ppy}) 3)(80 / 20 \mathrm{~nm}) / \mathrm{LiF}(1.4$ $\mathrm{nm}) / \mathrm{Al}(100 \mathrm{~nm})$ on a glass substrate. ITO was used as anode; NPD N,N '-Di(1-naphthyl)-N,N'-diphenyl-4,4'-diamine (also known as $\mathrm{N}, \mathrm{N}^{\prime}$-Bis (naphthalen-1-yl)-N, $\mathrm{N}^{\prime}$-bis(phenyl)benzidine (NPB)), serves as hole transport layer material; $\mathrm{TpBi}, 1,3,5$-tris( $\mathrm{N}$-phenylbenzimidizol-2-yl)benzene was used as a "Host" doped with a green phosphorescent dopant of $\operatorname{Ir}($ ppy)3, Tris[2-phenylpyridinato-C2,N]iridium(III), commonly known as a green phosphorescent emitter; LiF is used as electron injection layer material; and aluminium as a cathode. The whole OLED fabrication was performed in glovebox. The commercial barrier implemented was from OIKE Co. Ltd.

\section{Data availability}

The authors declare that [the/all other] data supporting the findings of this study are available within the paper [and its supplementary information files]. Any additional data that support the findings of this study are available from the corresponding author upon reasonable request.

\section{ACKNOWLEDGEMENTS}

We acknowledge funding via EPSRC-Innovate UK Grant (EP/M507751/1). B.C.B acknowledges funding from the European Union's Horizon 2020 research and innovation program under the Marie Skłodowska-Curie Grant Agreements 6562142DInterFOX. J.C.M. acknowledges support from the Austrian Science Fund (FWF, P25721-N20). We thank Philipp Braeuninger-Weimer, James Johnston and Sam Chan for their technical assistance and useful discussions.

\section{AUTHOR CONTRIBUTIONS}

A.A.S. and S.H. planned the experiments. A.A.S. and A.I.A. carried out growth. S.E., D.B. and A.A.S. performed Ca tests. S.E. and P.M. carried out OLED experiments. B.G. and P.B. contributed to traceability measurements. B.C.B. and J.C.M. performed plan-view TEM and SAED characterization. A.A.S. and S.H. wrote the manuscript with inputs from all authors.

\section{ADDITIONAL INFORMATION}

Supplementary information accompanies the paper on the npj $2 D$ Materials and Applications website (https://doi.org/10.1038/s41699-017-0037-z).

Competing interests: The authors declare no competing financial interests.

Publisher's note: Springer Nature remains neutral with regard to jurisdictional claims in published maps and institutional affiliations.

\section{REFERENCES}

1. Duncan, B., Urquhart, J. \& Roberts, S. Review of measurement and modelling of permeation and diffusion in polymers engineering. NPL Report DEPC MPR 012 (National Physical Laboratory, Middlesex, 2005).

2. Park, J.-S., Chae, H., Chung, H. K. \& Lee, S. I. Thin film encapsulation for flexible AM-OLED: a review. Semicond. Sci. Technol. 26, 34001 (2011).

3. Lewis, J. S. \& Weaver, M. S. Thin-film permeation-barrier technology for flexible organic light-emitting devices. IEEE J. Sel. Top. Quantum Electron. 10, 45-57 (2004).

4. Visweswaran, B. et al. Diffusion of water into permeation barrier layers. J. Vac. Sci. Technol. A 33, 31513 (2015).

5. Bird, D. et al. Production and measurement of large-area moisture barriers for electronic applications. 6th Electronic System-Integration Technology Conference (ESTC) 1-6 (IEEE, Grenoble, 2016).

6. Brewer, P. J., Goody, B. A., Kumar, Y. \& Milton, M. J. T. Accurate measurements of water vapor transmission through high-performance barrier layers. Rev. Sci. Instrum. 83, 75118 (2012)

7. Carcia, P. F., McLean, R. S., Reilly, M. H., Groner, M. D. \& George, S. M. Ca test of $\mathrm{Al}_{2} \mathrm{O}_{3}$ gas diffusion barriers grown by atomic layer deposition on polymers. Appl. Phys. Lett. 89, 3-6 (2006).
8. Park, C. Y., An, J. S., Jang, H. J., Lee, J. H. \& Choi, B. H. Growth behavior and improved water-vapor-permeation-barrier properties of 10-nm-thick single $\mathrm{Al}_{2} \mathrm{O}_{3}$ layer grown via cyclic chemical vapor deposition on organic light-emitting diodes. Org. Electron. 15, 1717-1723 (2014).

9. Jung, $\mathrm{H}$. et al. $\mathrm{Al}_{2} \mathrm{O}_{3}$ multi-density layer structure as a moisture permeation barrier deposited by radio frequency remote plasma atomic layer deposition. J. Appl. Phys. 115, 073502 (2014).

10. Xiao, W. et al. A flexible transparent gas barrier film employing the method of mixing ALD/MLD-grown $\mathrm{Al}_{2} \mathrm{O}_{3}$ and alucone layers. Nanoscale Res. Lett. 10, 130 (2011).

11. Nakano, Y., Yanase, T., Nagahama, T., Yoshida, H. \& Shimada, T. Accurate and stable equal-pressure measurements of water vapor transmission rate reaching the $10^{-6} \mathrm{~g} \mathrm{~m}^{-2}$ day $^{-1}$ range. Sci. Rep. 6, 35408 (2016).

12. Meyer, J. et al. $\mathrm{Al}_{2} \mathrm{O}_{3} / \mathrm{ZrO}_{2}$ Nanolaminates as ultrahigh gas-diffusion barriers - $\mathrm{A}$ strategy for reliable encapsulation of organic electronics. Adv. Mater. 21, 1845-1849 (2009).

13. Berry, V. Impermeability of graphene and its applications. Carbon 62, 1-10 (2013).

14. Miao, M., Nardelli, M. B., Wang, Q. \& Liu, Y. First principles study of the permeability of graphene to hydrogen atoms. Phys. Chem. Chem. Phys. 15, 16132-16137 (2013).

15. Tsetseris, L. \& Pantelides, S. T. Graphene: An impermeable or selectively permeable membrane for atomic species. Carbon 67, 58-63 (2014).

16. Hofmann, S., Braeuninger-Weimer, P. \& Weatherup, R. S. CVD-enabled graphene manufacture and technology. J. Phys. Chem. Lett. 6, 2714-2721 (2015).

17. $\mathrm{Wu}, \mathrm{T}$. et al. Fast growth of inch-sized single-crystalline graphene from a controlled single nucleus on Cu-Ni alloys. Nat. Mater. 15, 43-47 (2015).

18. Yan, Z., Peng, Z. \& Tour, J. M. Chemical vapor deposition of graphene single crystals. Acc. Chem. Res. 47, 1327-1337 (2014).

19. Choi, K. et al. Reduced water vapor transmission rate of graphene gas barrier films for flexible organic field-effect transistors. ACS Nano 9, 5818-5824 (2015).

20. Wirtz, C., Berner, N. C. \& Duesberg, G. S. Large-scale diffusion barriers from CVD grown graphene. Adv. Mater. Interfaces 2, 1-5 (2015).

21. Giesbers, A. J. M. et al. Defects, a challenge for graphene in flexible electronics. Solid State Commun. 229, 49-52 (2016).

22. Seo, T. H. et al. Tailored CVD graphene coating as a transparent and flexible gas barrier. Sci. Rep. 6, 24143 (2016).

23. Seo, H. K. et al. Laminated graphene films for flexible transparent thin film encapsulation. ACS Appl. Mater. Interfaces 8, (14725-14731 (2016).

24. Kim, H. W. et al. Selective gas transport through few-layered graphene and graphene oxide membranes. Science 342, 91-95 (2013).

25. Su, Y. et al. Impermeable barrier films and protective coatings based on reduced graphene oxide. Nat. Commun. 5, 4843 (2014)

26. O'Hern, S. C. et al. Nanofiltration across defect-sealed nanoporous monolayer graphene. Nano Lett. 15, 3254-3260 (2015).

27. Van Lam, D. et al. Healing defective CVD-graphene through vapor phase treatment. Nanoscale 6, 5639 (2014).

28. Aria, A. I. et al. Parameter space of atomic layer deposition of ultrathin oxides on graphene. ACS Appl. Mater. Interfaces 8, 30564-30575 (2016).

29. Aria, A. I. et al. Time evolution of the wettability of supported graphene under ambient air exposure. J. Phys. Chem. C 120, 2215-2224 (2016).

30. Nam, T. et al. A composite layer of atomic-layer-deposited $\mathrm{Al}_{2} \mathrm{O}_{3}$ and graphene for flexible moisture barrier. Carbon 116, 553-561 (2017).

31. Alexander-Webber, J. A. et al. Hysteresis-free encapsulated CVD graphene transistors via interface engineering of atomic layer deposited oxide. 2D Mater. 4, 1-9 (2016).

32. Nakashima, Y., Shirai, T. \& Takai, C. Synthesis of aluminum oxycarbide $\left(\mathrm{Al}_{2} \mathrm{OC}\right)$ by selective microwave heating. J. Ceram. Soc. Jpn. 124, 122-124 (2016).

33. Ryabkov, Y. I., Grass, V. E. \& Sitnikov, P. A. Synthesis of aluminum monooxycarbide. Russ. J. Gen. Chem. 72, 165-167 (2002).

34. $\mathrm{Wu}, \mathrm{Q}$. et al. Controllable poly-crystalline bilayered and multilayered graphene film growth by reciprocal chemical vapor deposition. Nanoscale 7, 10357-10361 (2015).

35. Zhou, $\mathrm{H}$. et al. Chemical vapour deposition growth of large single crystals of monolayer and bilayer graphene. Nat. Commun. 4, 2096 (2013).

36. Yao, Y. et al. Controlled growth of multilayer, few-layer, and single-layer graphene on metal substrates. J. Phys. Chem. C 115, 5232-5238 (2011).

37. Klumbies, H. et al. Thickness dependent barrier performance of permeation barriers made from atomic layer deposited alumina for organic devices. Org. Electron. 17, 138-143 (2015).

38. Nehm, F. et al. Breakdown and protection of ALD moisture barrier thin films. ACS Appl. Mater. Interfaces 7, 22121-22127 (2015).

39. Seethamraju, S. et al. Million-fold decrease in polymer moisture permeability by a graphene monolayer. ACS Nano 10, 6501-6509 (2016).

40. Braeuninger-Weimer, P., Brennan, B., Pollard, A. J. \& Hofmann, S. Understanding and controlling $\mathrm{Cu}$-catalyzed graphene nucleation: the role of 
impurities, roughness, and oxygen scavenging. Chem. Mater. 28, 8905-8915 (2016).

41. Chen, Y., Gong, X.-L. \& Gai, J.-G. Progress and challenges in transfer of large-area graphene films. Adv. Sci. 3, 1500343 (2016).

cc) Open Access This article is licensed under a Creative Commons Attribution 4.0 International License, which permits use, sharing, adaptation, distribution and reproduction in any medium or format, as long as you give appropriate credit to the original author(s) and the source, provide a link to the Creative
Commons license, and indicate if changes were made. The images or other third party material in this article are included in the article's Creative Commons license, unless indicated otherwise in a credit line to the material. If material is not included in the article's Creative Commons license and your intended use is not permitted by statutory regulation or exceeds the permitted use, you will need to obtain permission directly from the copyright holder. To view a copy of this license, visit http://creativecommons. org/licenses/by/4.0/.

(c) The Author(s) 2017 